\title{
Multiple free-radical scavenging capacity in serum
}

\author{
Shigeru Oowada, ${ }^{1, *}$ Nobuyuki Endo, ${ }^{2}$ Hiromi Kameya, ${ }^{3}$ Masashi Shimmei $^{4}$ and Yashige Kotake $^{5}$ \\ 'Division of Dialysis Center, Asao Clinic, Kawasaki, Kanagawa 215-0004, Japan \\ ${ }^{2}$ The Wakasa Wan Energy Research Center, Tsuruga, Fukui 914-0192, Japan \\ ${ }^{3}$ National Food Research Institute, Tsukuba, Ibaraki 305-8642, Japan \\ ${ }^{4}$ Radical Research Inc., Hino, Tokyo 191-0061, Japan \\ ${ }^{5}$ Oklahoma Medical Research Foundation, Oklahoma City, Oklahoma 73104 USA
}

(Received 19 September, 2011; Accepted 13 December, 2011; Published online 11 May, 2012)

\begin{abstract}
We have developed a method to determine serum scavengingcapacity profile against multiple free radical species, namely hydroxyl radical, superoxide radical, alkoxyl radical, alkylperoxyl radical, alkyl radical, and singlet oxygen. This method was applied to a cohort of chronic kidney disease patients. Each free radical species was produced with a common experimental procedure; i.e., uv/visible-light photolysis of free-radical precursor/sensitizer. The decrease in free-radical concentration by the presence of serum was quantified with electron spin resonance spin trapping method, from which the scavenging capacity was calculated. There was a significant capacity change in the disease group $(n=45)$ as compared with the healthy control group $(n=30)$. The percent values of disease's scavenging capacity with respect to control group indicated statistically significant differences in all free-radical species except alkylperoxyl radical, i.e., hydroxyl radical, $73 \pm 12 \% \quad(p=0.001) ; \quad$ superoxide radical, $158 \pm 50 \%$ $(p=0.001)$; alkoxyl radical, $121 \pm 30 \%(p=0.005)$; alkylperoxyl radical, $123 \pm 32 \%(p>0.1)$; alkyl radical, $26 \pm 14 \%(p=0.001)$; and singlet oxygen, $57 \pm 18 \%(p=0.001)$. The scavenging capacity profile was illustrated using a radar chart, clearly demonstrating the characteristic change in the disease group. Although the cause of the scavenging capacity change by the disease state is not completely understood, the profile of multiple radical scavenging capacities may become a useful diagnostic tool.
\end{abstract}

Key Words: multiple free radical, MULTIS, serum, ESR spin trapping, chronic kidney disease

$\mathrm{F}$ ree-radical scavenging capacity or antioxidant capacity in serum is thought to be an indicator of the body's defensive capacity against oxidative stress. ${ }^{(1,2)}$ Various methods have been proposed to evaluate the scavenging capacity in biological specimens. ${ }^{(3-8)}$ Most methods were categorized as indirect methods, where the probe's free-radical damage was indirectly monitored and the protection capacity of the specimen against the probe damage was evaluated. However, free radical species that was responsible for the damage has been rarely identified. In contrast, direct methods adopt electron spin resonance (ESR) technique to directly quantify the change in free radical concentration by the presence of the specimen, from which its free-radical scavenging capacity was calculated. ${ }^{(9)}$ Previously, using enzymatic superoxide generating systems, the scavenging capacity in biological fluid has been measured as superoxide dismutase (SOD)-like activity. ${ }^{(10)}$ In other studies, serum hydroxyl radical scavenging capacity has been measured with the use of the Fenton reaction; i.e., ferrous iron plus hydrogen peroxide as a free radical source. ${ }^{(9)}$ We used iron-free photochemical hydroxyl radical generating system and determined serum hydroxyl radical scavenging capacity in chronic kidney disease (CKD) patients. ${ }^{(11)}$ The results indicated that there was nearly a $40 \%$ decrease in hydroxyl radical scavenging capacity among the CKD group as compared with the healthy control group. Furthermore, in the same study, it was shown that the scavenging capacity was restored to the healthy level, immediately after hemodialysis treatment.

Obviously, the magnitude of free radical scavenging capacity is dependent on the kind of free radical species to be scavenged. ${ }^{(12)}$ However, a majority of previous direct-method studies concerned hydroxyl radical or superoxide radical. The significance of the scavenging capacity change in hydroxyl and superoxide radicals remains unclear because the mechanism by which these species may be causal to the disease is largely unknown. Moreover, free radical species that is involved in biological activity is not necessarily limited to hydroxyl or superoxide radical. For example, hepatotoxic carbon tetrachloride has been shown to produce trichloromethyl and other free radicals in vivo and in vitro. ${ }^{(13)}$ Thus, we believe that the knowledge on the antioxidant capacity against various free radical species may expand understanding of the change in the antioxidant capacity in the specific disease.

In the scavenging capacity measurement, it has been difficult to establish dependable free radical sources, especially when the free radical is short-lived. Stable free radical such as 2,2-diphenyl-1pycrylhydrazyl (DPPH) has been used in biological samples; ${ }^{(14)}$ however, DPPH is hardly related to biological systems. In situ photolysis of the free radical precursor in the ESR sample-cavity has been shown to be a convenient means to produce a finite amount of free radicals if the light-intensity and illumination-time are well-controlled. ${ }^{(15)}$ In this study, by selecting appropriate free radical precursors or photo-sensitizers, six different free radical species, namely hydroxyl radical, superoxide radical, alkoxyl radical, alkylperoxyl radical, alkyl radical, and singlet oxygen were produced with the common photolytic procedures. Produced free radicals were identified and quantified with ESR spin trapping method. ${ }^{(15)}$ In the presence of serum, the ESR signal intensity was decreased, depending on the magnitude of its scavenging capacity against the specific free radical. ${ }^{(11,12)}$ Thus, the objective of this study was to establish a method to determine scavenging capacities against multiple free radical species and seek for the possibility of using this method for medical purposes.

\section{Materials and Methods}

Materials and equipment. Light sources, illumination times, precursors and photo-sensitizers used in this study are listed in Table 1. Hydrogen peroxide, riboflavin, 2,2'-azobis (2-amidino-

*T whom correspondence should be addressed. E-mail: s2oowada@nifty.com 
Table 1. Photolytic production methods of multiple free radical species

\begin{tabular}{|c|c|c|c|c|c|c|c|}
\hline Free Radical & Precursor/Sensitizer & Light source $^{a}$ & Filter & $\begin{array}{l}\text { Wavelength } \\
\text { range }\end{array}$ & $\begin{array}{l}\text { Illumination } \\
\text { period }\end{array}$ & Spin trap & Reference \\
\hline$\cdot \mathrm{OH}$ & hydrogen peroxide $(10 \mathrm{mM})$ & RUVF-203SR & none & $300-400 \mathrm{~nm}$ & $5 \mathrm{~s}$ & CYPMPO & {$[15]$} \\
\hline $\mathrm{O}_{2}{ }^{\cdot-}$ & riboflavin $(20 \mu \mathrm{M})$ & RUVF-203SR & band-path & $500-600 \mathrm{~nm}$ & $60 \mathrm{~s}$ & CYPMPO & {$[16,17]$} \\
\hline$t$-BuO & AAPH (1 mM) & RUVF-203SR & none & $300-400 \mathrm{~nm}$ & $5 \mathrm{~s}$ & CYPMPO & [7] \\
\hline$t$-BuOO & $t$-butylhydro-peroxide (10 mM) & RUVF-203SR & none & $300-400 \mathrm{~nm}$ & $5 \mathrm{~s}$ & CYPMPO & {$[18]$} \\
\hline $\mathrm{CH}_{3} \cdot$ & $\mathrm{H}_{2} \mathrm{O}_{2}(100 \mathrm{mM})+$ DMSO $(10 \mathrm{mM})$ & RUVF-203SR & none & $300-400 \mathrm{~nm}$ & $5 \mathrm{~s}$ & CYPMPO & [19] \\
\hline${ }^{1} \Delta \mathrm{O}_{2}$ & rosebengal $^{\mathrm{b}}(200 \mu \mathrm{M})$ & RUVF-203SR & band-path & $500-600 \mathrm{~nm}$ & $60 \mathrm{~s}$ & 4-OH-TEMP & {$[20]$} \\
\hline
\end{tabular}

a RUVF-203SR illuminator delivers UV-visible light from a $200 \mathrm{~W}$ medium pressure $\mathrm{Hg} / \mathrm{Xe}$ arc to the ESR cavity via the quartz optical-guide. Light in-

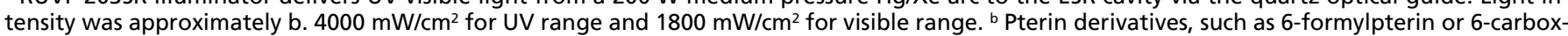
ylpterin can be used instead of rosebengal. ${ }^{(21)}$

propane) hydrochrolide (AAPH), $t$-butyl hydroperoxide, dimethyl sulfoxide (DMSO), rosebengal, and 4-hydroxy-2,2,6,6-tetramethylpiperidine (4-OH-TEMP) were purchased from Tokyo Chemical Industry (Tokyo, Japan) and used as received. Buffers and biochemical reagents were obtained from Wako Pure Chem. Ind. Ltd. (Osaka, Japan). The spin trap CYPMPO was obtained from Radical Research Inc. (Hino, Japan). ${ }^{(22)}$

UV light source was a $200 \mathrm{~W}$ medium-pressure mercury/xenon arc (RUVF-203SR UV illuminator, Radical Research Inc.), where UV-visible light was guided through a quartz light-guide into the ESR sample cavity. ESR spectrometer employed was a JEOL FR-80 (Akishima, Japan) equipped with $100 \mathrm{kHz}$ field modulation and a WIN-RAD operation software (Radical Research Inc.). Typical spectrometer settings were: field modulation width $0.1 \mathrm{mT}$; microwave power $6 \mathrm{~mW}$; field scan width and rate $\pm 7.5 \mathrm{mT} / 2 \mathrm{~min}$; time constant $0.1 \mathrm{~s}$.

Human subject. All human subject procedures were conducted with individual consent form and strictly following the protocol that had been approved by Asao Clinic Human Subject Use Committee. Blood was obtained from chronic kidney disease (CKD) patients undergoing stable maintenance hemodialysis (HD) $(n=45 ; 20$ females and 25 males; average age $65 \pm 13$; average HD durations $110 \pm 82$ months) and healthy volunteers $(n=30 ; 20$ females and 10 males; average age $35 \pm 15)$. The causes of CKD were as follows; 26 patients had chronic glomerulonephritis, 7 had diabetic nephropathy and 12 had nephrosclerosis. Serum was separated and stored in the $-30^{\circ} \mathrm{C}$ freezer until use. HD patients' blood was collected before hemodialysis.

Methods of free radical production. Each free radical species was produced via in situ illumination of UV/visible light from an RUVF-203SR UV illuminator to a buffer solution of corresponding free radical precursor or sensitizer. Table 1 shows the list of precursors/sensitizers and the detailed conditions for the photochemical free radical production for all six species along with previous publications for those production methods. ${ }^{(15-21)}$ For example, hydroxyl radical was produced by illuminating $5 \mathrm{~s}$ un-filtered UV light from the illuminator to a sodium phosphate buffer $(80 \mathrm{mM})$ solution of hydrogen peroxide $(10 \mathrm{mM})$ and CYPMPO $(10 \mathrm{mM})$, immediately followed by the recording of the ESR spectrum.

Measurement of serum free radical scavenging capacity. In the absence of serum, the signal intensity $\mathrm{I}_{0}$ of the selected ESR line was recorded, and in the presence of serum, the intensity I was again recorded. Using $\mathrm{I}_{0}$ and $\mathrm{I}$, the free radical scavenging capacity $k_{\text {serum }} / k_{\text {CYPMPO }}$ was calculated according to the following equation: ${ }^{(11,12)}$

$$
k_{\text {serum }} / k_{\text {CYPMPO }}=[\text { Serum }]_{0} /[\mathrm{ST}]_{0} /\left(\mathrm{I}_{0} / \mathrm{I}-1\right)
$$

where $[\text { Serum }]_{0}$ denotes the percent concentration of serum divided by 100 , and $[\mathrm{ST}]_{0}$ denotes CYPMPO concentration in $\mathrm{mM}$ unit. Therefore, $k_{\text {serum }} / k_{\text {CYPMPO }}$ was calculated in the $\mathrm{mM}$ CYPMPO equivalent unit. Typically, scavenging capacity was determined in 5 or $10 \%$ buffer solution of serum and the total volume of the sample solution was approximately $0.2 \mathrm{~mL}$.

Statistical analysis. At the designated conditions, three sample solutions were separately prepared and separate measurements were made. The raw data for $k_{\text {serum }} / k_{\text {CYPMPO }}$ were averaged and expressed with standard deviation (SD). Statistical test for the difference between the CKD group $(n=45)$ and the control group $(n=30)$ was performed using the Student's $t$ test.

\section{Results}

All free radical species except singlet oxygen were quantified with the ESR spin trapping method using CYPMPO as a spin trap. CYPMPO has been shown to have lower toxicity and better superoxide trapping performance in biological systems than similar spin traps. ${ }^{(22-24)}$ The spin-trapping reaction to capture the free radical $\mathrm{R}$ by CYPMPO proceeds as follows:

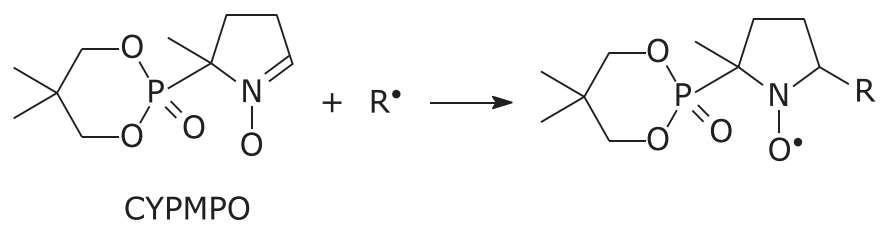

The ESR spectra of the spin adducts (the product in the above equation) for ${ }^{\circ} \mathrm{OH}, \mathrm{O}_{2}{ }^{-}, \mathrm{RO}^{\circ}, \mathrm{ROO}^{\circ}$, and $\mathrm{R}$ are shown in Fig. $1 \mathrm{~A}-\mathrm{E}$. Each spectrum was assigned to corresponding radical adduct and the hyperfine coupling constants (HFCC) for the ESR spectra were determined with computer spectrum simulation using an ESR simulation software in WIN-RAD system software (Table 2). Singlet oxygen was produced by illuminating visible light to the solution containing the photo-sensitizer rosebengal (Table 1). Singlet oxygen reacts with 4-OH-TEMP, forming the TEMPOL radical (4-hydroxy-2,2,6,6-tetramethylpiperidine-1-oxyl) that shows three line ESR spectrum (Fig. 1F) and the signal amplitude was proportional to the amount of singlet oxygen. A singlet-oxygen quencher, glutathione reduced form (GSH) was used to confirm the specificity. ${ }^{(25)}$

In the presence of serum, the signal amplitude decreased, from which the scavenging capacity (rate) was calculated with Eq. (1). Thus, Table 3 lists relative scavenging rates $\left(k_{\text {serum }} / k_{\text {spin trap }}\right)$ of the serum for all six free radical species. The radar chart in Fig. 2 illustrates percent scavenging-rate in the CKD group with respect to the control group. There were no differences of serum scavenging capacities among patients with DM or non-DM HD patients.

In Table 3 , the serum scavenging capacity $k_{\text {serum }} / k_{\text {CYPMPO }}$ is listed relative to that of $1 \mathrm{mM}$ CYPMPO, i.e., in the mM-CYPMPOeq unit. These numbers were converted into the unit equivalent to known pure scavengers, such as glutathione (GSH), superoxide 

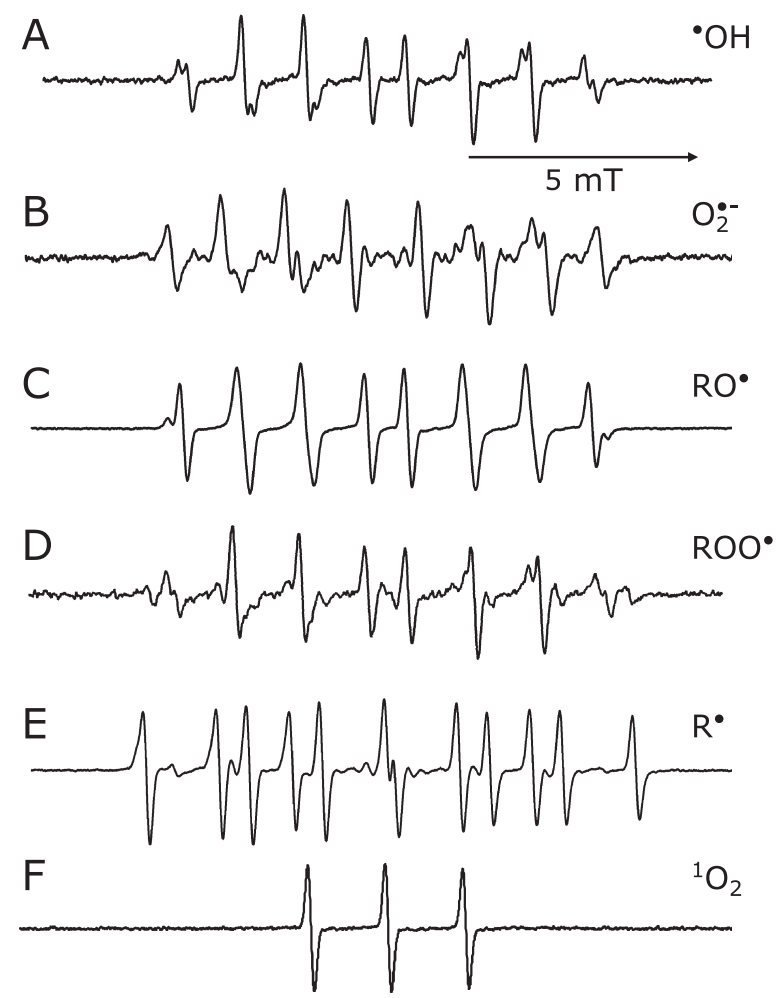

Fig. 1. ESR spectra of various spin adducts produced after photolysis of precursors/sensitizers (Table 1): A) hydroxyl radical adduct of CYPMPO; B) superoxide radical adduct of CYPMPO; C) t-butoxyl radical adduct of CYPMPO; D) t-butyl peroxyl radical adduct of CYPMPO; E) methyl radical adduct of CYPMPO; and F) TEMPOL radical formed after the reaction of singlet oxygen with 4-OH-TEMP. dismutase (SOD), trolox, and $\alpha$-lipoic acid (LA). Thus, we measured $k_{\mathrm{GSH}} / k_{\mathrm{CYPMPO}}$ for ${ }^{\circ} \mathrm{OH}$ and ${ }^{1} \Delta \mathrm{O}_{2}, \quad(=0.045$ and 0.28 , respectively), $k_{\text {SOD }} / k_{\text {CYPMPO for }} \mathrm{O}_{2}{ }^{--}(=0.0237), k_{\text {TROLOX }} / k_{\text {CYPMPO, for }}$ $\mathrm{RO}^{*}(=1.11)$, and $k_{\mathrm{LA}} / k_{\mathrm{CYPMPO}}$ for $\mathrm{ROO}^{*}(=0.105)$. These conversion factors were used to calculate $k_{\text {serum }} / k_{\text {SCAVENGER }}$ and the results were listed in the bottom panel of Table 3 .

\section{Discussion}

In the present study, we propose the multiple free-radical scavenging method (MULTIS) to measure serum scavenging capacities against six biologically relevant free radical species. The advantage of this method is to use the same experimental procedures to produce all six different free radical species. Briefly, the photolytic degradations of precursors were used in the case of hydroxyl, alkoxyl, and alkylperoxyl radicals. Alkyl (methyl) radical was produced from the photolysis of dimethyl sulfoxide (DMSO) plus hydrogen peroxide. ${ }^{(19)}$ Photo-sensitizers, riboflavin and rosebengal were used for the production of superoxide radical and singlet oxygen, respectively. Unlike other methods of free radical generation, all the six reactions would occur only when UV or visible light was turned on and their signal-intensities/ concentrations grew proportional to the illumination time at least in the early stage of illumination. Such facile handling of the reaction systems clearly differentiates this method from other free-radical generating systems like the hypoxanthine-xanthine oxidase system, the Fenton reaction system, or the fluorescencebased ORAC method. ${ }^{(4)}$

The results indicated that there were statistically significant shifts in the profile of the CKD group from the control group, except alkylperoxyl radical (Abstract, Table 3, and Fig. 2). Such changes are visibly obvious in radar chart illustration shown in Fig. 2. Scavenging capacity decreases in hydroxyl radical $(73 \pm 12 \%$ with respect to healthy control group), methyl radical $(26 \pm 14 \%)$ and singlet oxygen $(57 \pm 18 \%)$ were eminent. It is of interest to note that slight but statistically significant increases of the

Table 2. Hyperfine coupling constants of spin adducts.

\begin{tabular}{|c|c|c|c|c|}
\hline \multirow{2}{*}{ Free Radical } & \multicolumn{3}{|c|}{$\mathrm{HFCC} / \mathrm{mT}$} & \multirow{2}{*}{ Assignment } \\
\hline & Ан & $A_{N}$ & Ap & \\
\hline \multirow[t]{2}{*}{ Hydroxyl, $\bullet \mathrm{OH}$} & 1.37 & 1.37 & 4.88 & CYPMPO-OH ${ }^{a}$ \\
\hline & 1.23 & 1.35 & 4.70 & CYPMPO-OH ${ }^{a}$ \\
\hline \multirow[t]{2}{*}{ Superoxide, $\mathrm{O}_{2}{ }^{-}$} & 1.11 & 1.26 & 5.25 & $\mathrm{CYPMPO}-\mathrm{O}_{2}^{-\mathrm{b}}$ \\
\hline & 1.04 & 1.27 & 5.10 & $\mathrm{CYPMPO}-\mathrm{O}_{2}{ }^{-\mathrm{b}}$ \\
\hline$t$-Butoxyl, $t$-BuO•(Alkoxyl) & 1.24 & 1.36 & 4.80 & CYPMPO-OBuc \\
\hline$t$-Butoxyl, $t$-BuOO* (Alkylperoxyl) & 1.35 & 1.45 & 5.05 & CYPMPO-OOBu $^{d}$ \\
\hline Methyl, $\mathrm{CH}_{3} \cdot($ Alkyl) & 2.05 & 1.51 & 4.90 & CYPMPO-CH 3 \\
\hline Singlet Oxygen, ${ }^{1} \Delta \mathrm{O}_{2}$ & & 1.50 & & TEMPOL radicale \\
\hline
\end{tabular}

a Diastereomers. (14) b Diastereomers. (14) c Actual structure of $\mathrm{BuO}^{*}$ is $\mathrm{H}_{2} \mathrm{~N}(\mathrm{HN}) \mathrm{C}-\mathrm{C}\left(\mathrm{CH}_{3}\right)_{2}-\mathrm{O}^{*} \cdot{ }^{\text {(7) d }}$ Actual structure of BuOO*

is $\left(\mathrm{CH}_{3}\right)_{3} \mathrm{C}-\mathrm{OO}$. e TEMPOL radical $=4$-hydroxy-2,2,6,6-tetramethylpiperidine-1-oxyl radical.

Table 3. Relative scavenging capacity of the serum as measured for multiple free radical species

\begin{tabular}{|c|c|c|c|c|c|c|}
\hline & \multicolumn{6}{|c|}{ Free Radical } \\
\hline & $\cdot \mathrm{OH}$ & $\mathrm{O}_{2} \cdot-$ & $\mathrm{RO}^{\bullet}$ & ROO• & $\mathrm{R}^{\bullet}$ & ${ }^{1} \Delta \mathrm{O}_{2}$ \\
\hline & \multicolumn{5}{|c|}{$k_{\text {serum }} / k_{\text {CYPMPO }}$} & $k_{\text {serum }} / k_{\text {TEMP }}$ \\
\hline CKD $(n=45)$ & $0.31 \pm 0.05$ & $0.38 \pm 0.21$ & $1.25 \pm 0.31$ & $0.16 \pm 0.05$ & $0.09 \pm 0.05$ & $6.94 \pm 2.25$ \\
\hline percent vs control & $73 \pm 12 \%$ & $158 \pm 50 \%$ & $121 \pm 30 \%$ & $123 \pm 32 \%$ & $26 \pm 14 \%$ & $57 \pm 18 \%$ \\
\hline \multirow[t]{2}{*}{ healthy control $(n=30)$} & $0.42 \pm 0.12$ & $0.24 \pm 0.07$ & $1.03 \pm 0.07$ & $0.13 \pm 0.04$ & $0.35 \pm 0.14$ & $12.20 \pm 2.07$ \\
\hline & $\begin{array}{c}k_{\text {serum }} / k_{\mathrm{GSH}} \\
m M-G S H e q\end{array}$ & $\begin{array}{c}k_{\text {serum }} / k_{\text {sod }} \\
\text { U/ml-SODeq }\end{array}$ & $\begin{array}{c}k_{\text {serum}} / k_{\text {TROLOX }} \\
m M-T R O L O X e q\end{array}$ & $\begin{array}{c}k_{\text {serum }} / k_{\mathrm{LA}} \\
m M-\alpha-L A^{\mathrm{a} e q}\end{array}$ & $\begin{array}{c}k_{\text {serum }} / k_{\text {CYPMPO }} \\
m M-C Y P M P O^{\text {b eq }}\end{array}$ & $\begin{array}{c}k_{\text {serum}} / k_{\mathrm{GSH}} \\
\mu M-G S H e q\end{array}$ \\
\hline CKD $(n=45)$ & $6.8 \pm 1.1$ & $16.0 \pm 6.0$ & $1.13 \pm 0.28$ & $1.52 \pm 0.52$ & $0.09 \pm 0.05$ & $24.8 \pm 7.9$ \\
\hline healthy control $(n=30)$ & $9.4 \pm 2.8$ & $10.1 \pm 1.9$ & $0.93 \pm 0.06$ & $1.24 \pm 0.47$ & $0.35 \pm 0.14$ & $43.6 \pm 7.3$ \\
\hline
\end{tabular}

Top panel: Scavenging capacities of the serum are calculated relative to that of CYPMPO ( $k_{\text {serum}} / k_{\text {cyPMPo in }} \mathrm{mM}-\mathrm{CYPMPO}$ eq unit). For singlet oxygen, its quencher 4-OH-TEMP was used as a detection reagent and the capacity is listed as $k_{\text {serum }} / k_{\text {TEMP. }}$. Bottom panel: Scavenging capacities are converted into the equivalent units to pure scavengers. ${ }^{a} \alpha$-lipoic acid. ${ }^{b}$ There is no known scavengers for alkyl radical (R). 


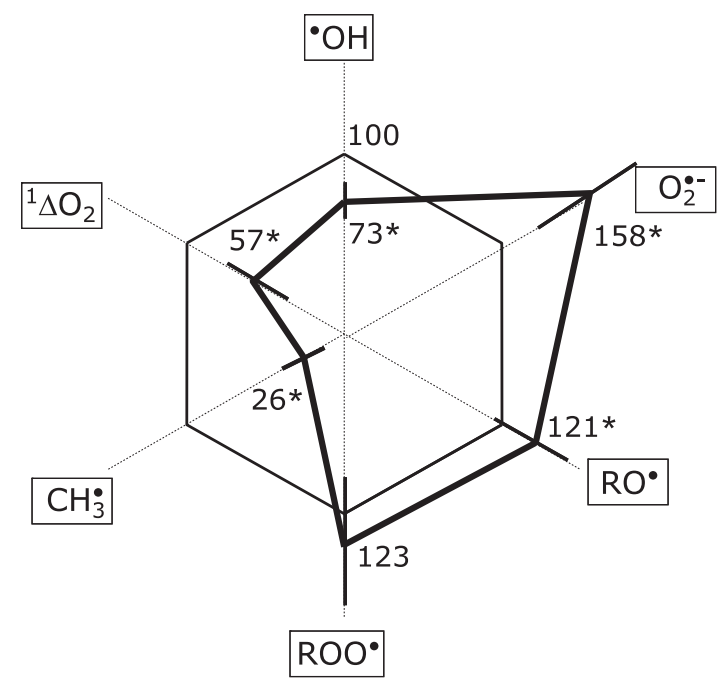

Fig. 2. A radar chart illustration of the relative scavenging capacity data listed in Table 3. Percent changes in the capacity of the CKD group (thick lines) are shown with respect to the control group. Error bars are shown in each free radical species and the numbers marked with asterisk showed statistically significant difference from the control group.

scavenging capacities in the CKD group were found in superoxide radical, t-butoxyl radical, and t-butylperoxyl radical. Although disease conditions usually incapacitate defensive mechanism against oxidative stress the scavenging-capacity increase may indicate that certain defensive mechanisms had been induced and enhanced in CKD. When more data will be available for various diseases such illustration would be potentially useful in judging the state of the specific disease.

It is quite possible that serum antioxidant capacity is readily modified by the disease state. Recently Chen et al. ${ }^{(26)}$ reported that the scavenging capacity changes in the plasma of CKD patients $(n=20)$ against singlet-oxygen chemiluminescence, hypochlorite, hydrogen peroxide, and hydroxyl radical (FRAP assay). Scavenging capacities for hydrogen peroxide and singlet oxygen have been shown to decrease by approximately $20 \%$ in pre-hemodialytic plasmas. But, all parameters showed significant decreases in the post-hemodialytic plasmas. Although there was no clear interpretation for these changes the approach that was adopted in that study was in line with the present study. However, the identification of the mechanism by which serum or plasma antioxidant capacity is modified by the disease would be difficult and time-consuming and could be a futile effort. There must be enormous number of factors that can influence serum free radical scavenging capacity. Even a slight change in water- or proteincontent in the serum would have caused the shift in the scavenging capacity. ${ }^{(10)}$ Instead of pursuing the cause of shift in the scavenging capacity, we took a practical standpoint and directed this work for future applications. A remarkable radar chart display in Fig. 2 seems to support the direction.

In conclusion, we have proposed the MULTIS method to demonstrate scavenging-profile deviation in a cohort of CKD patients. It is likely that MULTIS profile would be disease specific, thus this method potentially become a tool for diagnosis or prognosis.

\section{Conflict of Interest}

No potential conflicts of interest were disclosed.

\section{Abbreviations}

\begin{tabular}{|c|c|}
\hline CKD & chronic kidney disease \\
\hline CYPMPO & $\begin{array}{l}\text { 5-(2,2-dimethyl-1,3-propoxy cyclophosphoryl)-5- } \\
\text { methyl-1-pyrroline N-oxide }\end{array}$ \\
\hline DM & diabetes mellitus \\
\hline DMSO & dimethyl sulfoxide \\
\hline ESR & electron spin resonance \\
\hline GSH & glutathione reduced form \\
\hline HD & hemodialysis \\
\hline HFCC & hyperfine coupling constants \\
\hline$\alpha$-LA & $\alpha$-lipoic acid \\
\hline MULTIS & multiple-radical scavenging \\
\hline 4-OH-TEMP & 4-hydroxy-2,2,6,6-tetramethylpiperidine \\
\hline ORAC & oxygen radical absorbance capacity \\
\hline & geroxide dismutase \\
\hline
\end{tabular}

\section{References}

1 McCord JM, Wong K. Oxygen free radicals and tissue damage. Excerpta Medica 1979; 65: 343-351.

2 Halliwell B, Gutteridge JMC. Free radicals in biology and medicine. Tokyo: Japan Scientific Societies Press, 1988.

3 Glazer AN. Phycoerythrin fluorescence-based assay for reactive oxygen species. Methods Enzymol 1990; 186: 161-168.

4 Cao G, Alessio HM, Cutler RG. Oxygen-radical absorbance capacity assay for antioxidants. Free Radic Biol Med 1993; 14: 303-311.

5 Benzie IF, Strain JJ. The ferric reducing ability of plasma (FRAP) as a measure of "antioxidant power": the FRAP assay. Anal Biochem 1996; 239: 70-76.

6 Wayner DD, Burton GW, Ingold KU, Locke S. Quantitative measurement of the total, peroxyl radical-trapping antioxidant capability of human blood plasma by controlled peroxidation. The important contribution made by plasma proteins. FEBS Lett 1985; 187: 33-37.

7 Noda Y, Anzai K, Mori A, Kohno M, Shinmei M, Packer L. Hydroxyl and superoxide anion radical scavenging activities of natural source antioxidants using the computerized JES-FR30 ESR spectrometer system. Biochem Mol Biol Int 1997; 42: 35-44.

8 Prior RL, Cao G. In vivo total antioxidant capacity: comparison of different analytical methods. Free Radic Biol Med 1999; 27: 1173-1181.

9 Tanigawa T. Determination of hydroxyl radical scavenging activity by electron spin resonance. J Kyoto Pref Univ Med 1990; 99: 133-143.

10 Hiramatsu M, Kohno M, Edamatsu R, Mitsuta K, Mori A. Increased super-

oxide dismutase activity in aged human cerebrospinal fluid and rat brain determined by electron spin resonance spectrometry using the spin trap method. J Neurochem 1992; 58: 1160-1164.

11 Endo N, Oowada S, Sueishi Y, et al. Serum hydroxyl radical scavenging capacity as quantified with iron-free hydroxyl radical source. J Clin Biochem Nutr 2009; 45: 193-201.

12 Kohri S, Fujii H, Oowada S, et al. An oxygen radical absorbance capacitylike assay that directly quantifies the antioxidant's scavenging capacity against AAPH-derived free radicals. Anal Biochem 2009; 386: 167-171.

13 McCay PB, Lai EK, Poyer JL, DuBose CM, Janzen EG. Oxygen- and carbon-centered free radical formation during carbon tetrachloride metabolism. Observation of lipid radicals in vivo and in vitro. J Biol Chem 1984; 259 . 2135-2143.

14 Hiramatsu M, Liu J, Edamatsu R, Ohba S, Kadowaki D, Mori A. Probucol scavenged 1,1-diphenyl-2-picrylhydrazyl radicals and inhibited formation of thiobarbituric acid reactive substances. Free Radic Biol Med 1994; 16: 201216.

15 Kotake Y, Janzen EG. Decay and fate of the hydroxyl radical adduct of $\alpha$ phenyl $N$-tert-butylnitrone in aqueous media. J Am Chem Soc 1991; 113: 9503-9506.

16 Kim H, Kirschenbaum LJ, Rosenthal I, Riesz P. Photosensitized formation of ascorbate radicals by riboflavin: an ESR study. Photochem Photobiol 1993; 57: 777-784.

17 Prolla TA, Mehlhorn RJ. A photochemical system for generating free radicals: 
superoxide, phenoxyl, ferryl and methyl. Free Radic Res Commun 1990; 9: $135-146$.

18 Janzen EG, Blackburn BJ. Detection and identification of short lived free radicals by an electron spin resonance trapping techniques. $\mathrm{J} \mathrm{Am} \mathrm{Chem} \mathrm{Soc}$ 1969; 91: 4481-4490.

19 Burkitt MJ, Mason RP. Direct evidence for in vivo hydroxyl-radical generation in experimental iron overload: an ESR spin-trapping investigation. Proc Natl Acad Sci USA 1991; 88: 8440-8444.

20 Zang LY, van Kuijk FJ, Misra BR, Misra HP. The specificity and product of quenching singlet oxygen by 2,2,6,6-tetramethylpiperidine. Biochem Mol Biol Int 1995; 37: 283-293.

21 Thomas AH, Lorente C, Capparelli AL, Martínez CG, Braun AM, Oliveros E. Singlet oxygen $\left({ }^{1} \Delta \mathrm{g}\right)$ production by pterin derivatives in aqueous solutions. Photochem Photobiol Sci 2003; 2: 245-250.

22 Kamibayashi M, Oowada S, Kameda H, et al. Synthesis and characterization of a practically better DEPMPO-type spin trap, 5-(2,2-dimethyl-1,3-propoxy cyclophosphoryl)-5-methyl-1-pyrroline N-oxide (CYPMPO). Free Radic Res 2006; 40: 1166-1172.

23 Saito K, Takahashi M, Kamibayashi M, Ozawa T, Kohno M. Comparison of superoxide detection abilities of newly developed spin traps in the living cells. Free Radic Res 2009; 43: 668-676.

24 Rai K, Matsui H, Kaneko T, et al. Lansoprazole inhibits mitochondrial superoxide production and cellular lipid peroxidation induced by indomethacin in RGM1 cells. J Clin Biochem Nutr 2011; 49: 25-30.

25 Devasagayam TP, Sundquist AR, Di Mascio P, Kaiser S, Sies H. Activity of thiols as singlet molecular oxygen quenchers. J Photochem Photobiol B 1991; 9: $105-116$.

26 Chen TS, Liou SY, Chang YL. Chemiluminescent analysis of plasma antioxidant capacity in uremic patients undergoing hemodialysis. Ren Fail 2008; 30: $843-847$. 